J. Clin. Chem. Clin. Biochem.

Vol. 15, 1977, pp. $121-130$

\title{
Enzymatische Bestimmung des Gesamtcholesterins mit dem Greiner Selective Analyzer (GSA-II)
}

\author{
Von K. Borner \\ Institut für Klinische Chemie und Klinische Biochemie (Direktor: Prof. Dr. H.-J. Dulce) der Freien Universität Berlin, \\ Klinikum Steglitz und
}

\section{S. Klose}

Fa. Boehringer Mannheim, Biochemica Werk Tutzing

(Eingegangen am 9. August 1976)

Zusammenfassung: Es wird eine vollenzymatische Methode zur Bestimmung des Gesamtcholesterins im Serum beschrieben, die sich u. E. besonders gut für diskrete vollmechanische Analysen-Geräte, u. a. auch für MehrkanalAnalysatoren, eignet. Die Methode verwendet die Enzyme Cholesterinesterase (EC 3.1.1.13), Cholesterinoxidase (EC 1.1.3.6) und Peroxidase (EC 1.11.1.7) mit 4-Aminophenazon und Phenol als Substrate in der Indikatorreaktion. Die Methode wurde an den Greiner Selective Analyzer GSA-II adaptiert. Die dafür kritischen Reaktions-Parameter wurden ausführlich geprüft.

Das fertige Reagenz ist im GSA II-Dosierer mindestens 1 Woche bei $4{ }^{\circ} \mathrm{C}$ stabil. Durch Weglassen der Cholesterinoxidase im Vergleichswert wird ein Probenleerwert und partieller Reagenzienleerwert bestimmt und vom Hauptwert abgezogen. Bei einem Meßbereich bis $10,4 \mathrm{mmol} / 1(4,0 \mathrm{~g} / \mathrm{l})$ wird das maximale Signal in 6 Minuten erreicht. Der Kalibrier-Faktor war 4 Monate lang stabil. Absolut-Messungen sind daher mit der Methode möglich. Die Präzision in Serie lag bei Konzentrationen von 2 bis $4 \mathrm{mmol} / \mathrm{l}$ zwischen 0,5 und 1,4\%. Die Präzision von Tag zu Tag lag für dieselben Kontrollseren bei 2,8; 2,0;2,7 und 2,0\% während 3 Monaten. Die Richtigkeitsprüfung ergab zufriedenstellende Ergebnisse.

Ascorbinsäure stört die Reaktion in normalen Konzentrationen nicht in nennenswertem Umfang. Keine Störung wurde bei in vitro Zusatz von Katalase oder Novaminsulfon beobachtet. Spektrale Interferenzen durch Bilirubin, Hämoglobin und Trübungen werden durch den Probenleerwert eliminiert. Der Vergleich von Ergebnissen mit der enzymatischen Methode von Roeschlau et al. (Z. Klin. Chem. Klin. Biochem. 12, 226 (1974)) ergab eine befriedigende Ubereinstimmung. Die Nachweisempfindlichkeit der Methode kann durch Ersatz des Phenols durch DihalogenPhenole um das 2,2fache gesteigert werden.

\section{Enzymatic determination of total cholesterol with the Greiner Selective Analyzer (GSA-II)}

Summary: A fully enzymatic method to determine total cholesterol in serum is described. The method appears especially suitable for adaptation to discrete mechanical analyzers either of the single channel or the multi-channel type. The method uses the enzymes cholesterol esterase (EC 3.1.1.13), cholesterol oxidase (EC 1.1.3.6) and peroxidase (EC 1.11.1.7) with 4-aminophenazone and phenol as substrates in the indicator reaction. The method was adapted to the Greiner Selective Analyzer GSA-II. For this purpose the critical parameters of the reaction were intensively examined.

The complete reagent is stable within the GSA II dispenser at $4{ }^{\circ} \mathrm{C}$ for at least 1 week. By omitting cholesterol oxidase in the blank reagent a sample blank and a partial reagent blank are obtained. Within a range up to $10.4 \mathrm{mmol} / \mathrm{l}(4.0 \mathrm{~g} / \mathrm{l})$ the maximum colour is developed within 6 minutes. The calibration factor was stable for 4 months. The method allows absolute measurements. At concentrations between 2 and $4 \mathrm{mmol} / \mathrm{l}$ within-batch precision ranged from 0.5 to $1.4 \%$. Precision from day to day for the same control sera amounted to $2.8 ; 2.0 ; 2.7$ and $2.0 \%$ for a period of 3 months. Examination of accuracy yielded satisfying results. 
Ascorbic acid in the physiological range did not alter results to a significant extent. Catalase or novaminesulfone added in vitro did not interfere. Optical interferences by bilirubin, hemoglobin or turbidity are compensated by a sample blank. A comparison of results with the enzymatic method of Roeschlau et al. (Z. Klin. Chem. Klin. Biochem. $12,226(1974))$ yielded satisfactory agreement.

The limits of detection of the present method can be lowered by a factor of 2.2 by replacing phenol by dihalogen phenols.

\section{Einführung}

Anläßlich einer 9 Monate dauernden Erprobung des Großautomaten Greiner Selective Analyzer (GSA-II) ergab sich die Notwendigkeit, eine für diesen Gerätetyp optimale Methode zur Bestimmung des Gesamtcholesterins zu entwickeln. Das in dieser Zeitschrift ausführlich beschriebene Gerät (1) bietet in der sogenannten EDV-Version erhebliche datentechnische Vorzüge, wie flexible direkte Probenidentifikation, selektive probenindividuelle Bearbeitung bei konstantem Datenausstoß und automatisches Erstellen vollständiger Ergebnissätze. Als diskretes Analysen-System zeigt der GSA-II jedoch analytische Limitierungen, die für viele Geräte dieser Klasse typisch sind:

1. Es sind keine Phasentrennungen möglich.

2. Eine einheitliche, allerdings wählbare Reaktionstemperatur, z. B. $37^{\circ} \mathrm{C}$.

3. Eine Begrenzung der maximalen Reaktionszeit, im Falle des GSA-II auf 9,6 Minuten.

4. Aggressive oder flüchtige Reagenzien sollten nicht eingesetzt werden.

5. Das Photometer erlaubt Messungen mit einer von 6 $\mathrm{Hg}$-Linien zwischen 334 und $577 \mathrm{~nm}$.

Da keine der zum Zeitpunkt der Erprobung - im Sommer 1975 - bekannten Methoden befriedigende Ergebnisse lieferte, wurde eine enzymatische Methode $(2,3)$ von uns an den GSA-Il angepaßt, wobei als Indikator-Reaktion zum Nachweis von Wasserstoffperoxid die zuerst von Trinder (4) angegebene Reaktion dient:

Cholesterin-Ester $+\mathrm{H}_{2} \mathrm{O} \underset{(\mathrm{EC} 3.1 .1 .13)}{\stackrel{\text { Cholesterinesterase }}{\longrightarrow}}+\begin{aligned} & \text { Cholesterin } \\ & + \text { Fettsäure }\end{aligned}$

Cholesterin $+\mathrm{O}_{2} \underset{\text { Cholesterinoxidase }}{\stackrel{\mathrm{EC} 1.1 .3 .6)}{\longrightarrow}} \Delta^{4}$-Cholestenon

$2 \mathrm{H}_{2} \mathrm{O}_{2}+4$-Aminophenazon + Phenol

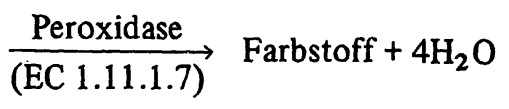

(Als Farbstoff wird das 4-( $p$-Benzochinon-monoimino)phenazon angegeben).

Die Methode sollte weiterhin einige für Großgeräte spezifische Anforderungen erfüllen, auf die weiter unten eingegangen wird.

\section{Geräte, Reagenzien, Methoden}

Geräte

1. Der Greiner Selective Analyzer (GSA-II) wurde in der sog. EDV-Version benutzt. Dabei stehen neben dem Grundgerät (1) ein Thermodrucker und ein Kartenstanzer zur Verfügung. Für jedes Ergebnis wird eine Karte gelocht. Die weitere Auswertung der Ergebnisse erfolgte mit dem Labor-Rechner des Chemischen Zentrallabors (Hewlett Packard 2100 A) unter Verwendung vorhandener Standard-Programme.

2. Die Kinetik der Reaktionen wurde mit einem Photometer Eppendorf mit Küvettenwechselaụtomatik und Schreiber bei $37^{\circ} \mathrm{C}$ gemessen.

3. Absorptionsspektren wurden mit einem registrierenden Spektralphotometer DMR 21 der Fa. C. Zeiß, Oberkochen, registriert.

Reagenzien, Standards

Als Fertig-Reagenz wurde die Automaten-Packung Nr. 15537 der Fa. Boehringer-Mannheim in modifizierter Form verwendet. Die substituierten Phenole waren zum Teil eine Gabe der Firma Boehringer, zum Teil wurden sie von der Firma MerckSchuchardt bezogen. Zur Kalibrierung u. ä. wurden die primären Standard-Lösungen Preciset Cholesterin und Precimat Cholesterin der $\mathrm{Fa}$. Boehringèr verwendet.

Handelsübliches Kontrollmaterial wurde, soweit gefriergetrocknet, unter Verwendung von geeichten Pipetten nach Vorschrift aufgelöst. Die Pipettierung wurde durch Wiegen kontrolliert. Die größte zugelassene Abweichung betrug $\pm 0,5 \%$ vom Sollwert.

\section{Analy tisches Verfahren}

\section{Arbeits-Reagenzien}

Die Originalvorschrift des Herstellers wurde dahingehend modifiziert, daß alle Substanzen mit Ausnahme des Enzyms Cholesterinoxidase zu einer Lösung angesetzt werden. Diese L,ösung wird anschhließend in 2 gleichgroße Teile geteilt. Der einen Hälfte wird die gesamte vorhandene Menge der Cholesterinoxidase hinzzugefügt (Haupt-Reagenz). Die andere Hälfte wird ohne Zusatz auf das gleiche Endvolumen verdünnt (LeerwertReagenz). Leerwert- und Hauptreagenz müssen aus einer Charge gleichzeitig angesetzt werden. Das Auffüllen der Reagenzien sollte zügig unter Vermeiden direkten Sonnenlichtes erfolgen. Nicht benutztes Reagenz wird in braunen Flaschen im Kühlschrank aufbewahrt. Die Konzentrationen im Test sind in Tabelle 1 angegeben. (Eine detaillierte Methodenbeschreibung steht auf Wunsch zur Verfügung.)

Bei den Versuchen mit substituierten Phenolen wurde die Einwaage des entsprechenden Phenols in jeweils $10 \mathrm{ml}$ Methanol p. A. gelöst und anstelle des in der Packung enthaltenen Phenols in je 1 Liter endgültiges Testgemisch eingefügt. Die Reagenzien der manuellen Methode wurden nach Vorschrift des Herstellers angesetzt und verweñdet.

\section{Analytisches Vorgehen}

Das analytische Vorgehen bèschreibt die Abbildung 1 in der vóm Hersteller des Gerätes standardisierten Form. Der Leerwert ist bei den beschriebenen Verfahren sowöhl ein Probenleerwert als 
Tab. 1. Testbedingungen: Konzentrationen im Ansatz sowie andere wichtige Parameter.

\begin{tabular}{|c|c|c|c|}
\hline $\begin{array}{l}\text { Reaktions- } \\
\text { parameter }\end{array}$ & & $\begin{array}{l}\text { GSA-II- } \\
\text { Verfahren }\end{array}$ & $\begin{array}{l}\text { manuelles } \\
\text { Verfahren }\end{array}$ \\
\hline $\begin{array}{l}\text { Temperatur } \\
\text { pH }\end{array}$ & $\left({ }^{\circ} \mathrm{C}\right)$ & $\begin{array}{l}37 \\
7.2\end{array}$ & $\begin{array}{l}\text { Raumte mperatur } \\
\quad 7.7\end{array}$ \\
\hline Reaktionszeit & $(\min )$ & 9,2 & $>15 \quad(30)$ \\
\hline $\begin{array}{l}\text { Probenverdünnung } \\
\text { Meßwellenlänge }\end{array}$ & & & $2 \stackrel{1: 103}{500(480-546)}$ \\
\hline $\begin{array}{l}\text { Kalium-Phosphat- } \\
\text { Puffer }\end{array}$ & $(\mathrm{mmol} / \mathrm{l})$ & 190 & 387 \\
\hline Phenol & $(\mathrm{mmol} / \mathrm{l})$ & 3,0 & 9,7 \\
\hline Methanol & $(\mathrm{mmol} / \mathrm{l})$ & 476 & 1790 \\
\hline 4-Aminophenazon & $(\mathrm{mmol} / \mathrm{l})$ & 1,81 & 0,97 \\
\hline $\begin{array}{l}\text { Hydroxypoly- } \\
\text { ethoxydodekan } \\
\text { (Thesit) }\end{array}$ & $(g / l)$ & $\cdot 1,9$ & 2,0 \\
\hline $\begin{array}{l}\text { Cholesterin- } \\
\text { esterase }\end{array}$ & $(U / 1)$ & 38 & 181 \\
\hline $\begin{array}{c}\text { Cholesterin- } \\
\text { oxidase }\end{array}$ & $(U / I)$ & 76 & 39 \\
\hline $\begin{array}{c}\text { Cholesterin- } \\
\text { oxidase }\end{array}$ & & Leerwerte: 0 & 0 \\
\hline Peroxidase & $(U / 1)$ & 1238 & 3802 \\
\hline Cholesterin & $(\mu \mathrm{mol} / \mathrm{l})$ & bis 103 & 128 \\
\hline Cholesterin & $(\mathrm{mg} / \mathrm{l})$ & bis 32 & 49 \\
\hline
\end{tabular}

\begin{tabular}{|c|l|c|c|c|c|}
\hline \multirow{2}{*}{\multicolumn{2}{|c|}{ REAGENT }} & TIME & \multicolumn{2}{c|}{ VOLUME } & \multicolumn{1}{c|}{ ASSAY } \\
\cline { 2 - 6 } & $\min \times 10$ & 1 & 11 & \\
\hline & S+DF $(X+100)$ & 100 & 120 & 120 \\
\hline R-1 & Reagenz & 92 & - & 2400 \\
\hline R-2 & Leerwert Reagenz & 81 & 2400 & \\
\hline & & & & \\
\hline & & & & \\
\hline & & & & \\
\hline & READING & 0 & 2520 & 2520 & \\
\hline
\end{tabular}

Abb. 1. Dosierschema für die Cholesterinbestimmung, PAPMethode, im GSA II.

Berechnung des Ergebnisses: $c=\Delta \mathrm{A} \cdot$ Faktor. Bei $546 \mathrm{~nm}$ beträgt der Faktor $12,63(\mathrm{~g} / \mathrm{l}) \mathrm{bzw}$. $32,71(\mathrm{mmol} / \mathrm{l})$.

Alternativ kann bei $492 \mathrm{~nm}$ gemessen werden.

auch ein partieller Reagenzien-Leerwert, der vom Hauptwert subtrahiert wird.

Die Richtigkeit der Dosierer wurde durch den sog. MaschinenTest mit $p$-Nitrophenol-Lösungen regelmäßig geprüft.

\section{Ergebnisse}

\section{Eigenschaften des Reagenz}

Das fertig angesetzte Reagenz ist bei $4^{\circ} \mathrm{C}$, d. h. im Analysengerät, mindestens 1 Woche stabil. Innerhalb von 4 Monaten wurde kein Keimwachstum in dẹ Dispensern beobachtet. Die Lichtempfindlichkeit ist unkritisch, wenn lange Belastungen durch intensives Sonnenlicht vermieden werden. Der Reagenzienleerwert steigt in beiden Reagenzien in 1-2 Wochen um die Absorptionsdifferenz 0,1 an, ohne Änderung der Signalhöhen. Die Löslichkeit aller Bestandteile bei $4{ }^{\circ} \mathrm{C}$ ist gut. Nach dem Ansetzen des Reagenz wird gelegentlich eine Aktivitätszunahme innerhalb der ersten 12 Stunden beobachtet. Anschließend ist die Aktivität mindestens 1 Woche lang unverändert stabil. Das Reagenz konnte aus Einzelsubstanzen rekonstruiert werden, war allerdings weniger stabil. - Der Preis pro Test beträgt unter dem angegebenen Verfahren DM 0,91 und bei Verwendung des Standard-Probenvolumens $(10 \mu \mathrm{l}) \mathrm{DM} 0,45$.

\section{Linearität, Meßbereich und Nachweisgrenze}

Bei Analyse primärer Standard-Lösungen (Preciset) von freiem Cholesterin ergeben sich lineare Bezugskurven bis $10,4 \mathrm{mmol} / \mathrm{l}(4,0 \mathrm{~g} / \mathrm{l})$. Gelegentlich finden sich jedoch bei Konzentrationen über $5,2 \mathrm{mmol} / \mathrm{l}(2,0 \mathrm{~g} / \mathrm{l})$ proportional höhere Absorptionen mit Abweichungen bis zu maximal $+7 \%$ bezogen auf die Steilheit der Bezugskurve bei $5,2 \mathrm{mmol} / \mathrm{l}$. Die Abweichungen traten besonders dann auf, wenn die Kalibrierproben einige Zeit offen standen. Gravimetrische Bestimmungen der Verdunstung von Preciset ergeben bei $1,0 \mathrm{ml}$ Probe in einem offenen Technicon-Gefäß einen Verdunstungsverlust von $2,4 \%$ in 60 Minuten. Der gleichzeitig bestimmte Verdunstungsverlust von Wasser betrug $1,5 \%$ in 60 Minuten.

Die Linearität der Gesamt-Reaktion wurde weiterhin an Verdünnungsreihen von Patientenseren mit hohem Cholesteringehalt überprüft (Abb. 2). Das Signal war mindestens bis zu $9 \mathrm{mmol} / \mathrm{l}$ linear. Bei höheren Konzentrationen waren die Absorptionen relativ kleiner. Die untere Nachweisgrenze beträgt $0,16 \mathrm{mmol} / 1$ (63 mg/l).

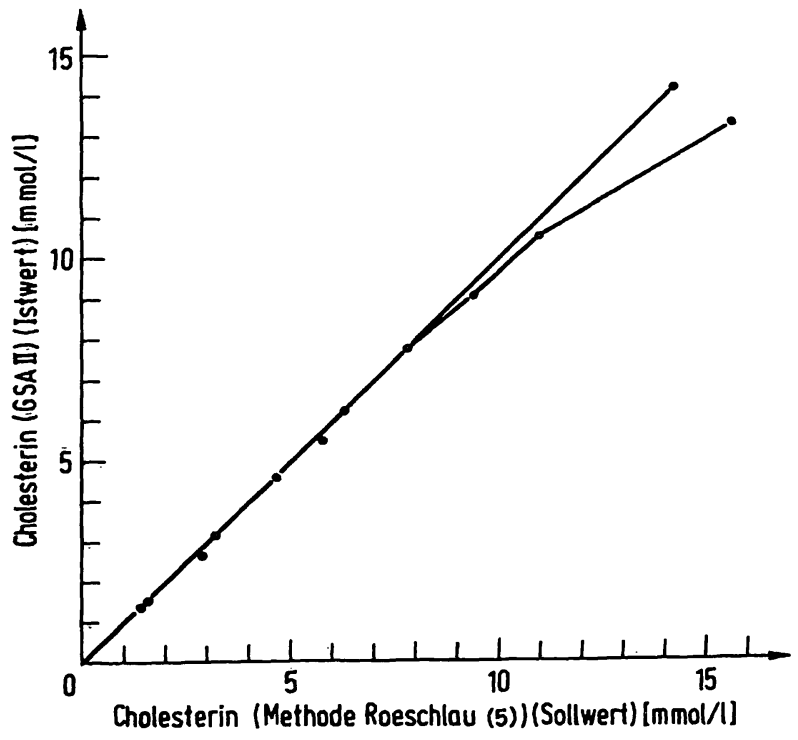

Abb. 2. Überprüfung der Linearität der Cholesterinbestimmung im GSA II mit Patienten-Proben. Die Sollwerte wurden nach Roeschlau (5) ermittelt. Die Proben wurden mit $9 \mathrm{~g} / \mathrm{l} \mathrm{NaCl}$ verdünnt. Jeder Punkt ist das Mittel einer Doppelbestimmung. 


\section{Kinetik der Reaktion}

Abbildung 3 zeigt den Reaktionsverlauf mit primären Standard-Lösungen von Cholesterin. Das Maximum der Farbentwicklung wird bei pH 7,2 (!) je nach Konzentration in 4-6 Minuten erreicht. Über dem Meßbereich liegende Konzentrationen erreichen ihr Farbmaximum erst später, u. U. erst nach der Meßzeit von 9 Minuten. Bei höheren Konzentrationen findet sich anschließend ein geringfügiger Absorptionsabfall von etwa $0,2 \%$ des Maximums pro Minute, der jedoch bei automatischer Messung unbedeutend ist und daher vernachlässigt werden darf. Um die Gesamt-Reaktion, d. h. einschließlich der Cholinesterase-Reaktion zu überprüfen, wurde bei etwa 30 Patientenseren mit Cholesterin-Konzentrationen zwischen 5 und $10 \mathrm{mmol} / \mathrm{l}$ die Farbentwicklung fortlaufend registriert. Dabei kommt es zum Teil zu verlangsamten Phasen im Reaktionsverlauf, was, aus dem Reaktionsschema und der Spezifität des Enzyms für verschiedene Ester verständlich ist. Das Maximum der Farbreaktion wurde jedoch fast immer in der vorgesehenen Meßzeit erreicht (Abb. 4). Die Farbe ist bei Patienten- und Kontrollseren etwas stabiler als bei primären Standard-Lösungen.

Bei hohen Triglycerid-Konzentrationen mit sichtbarer Trübung der Probe kann eine stark verzögerte Reaktion auftreten, was zu einem falsch niedrigen Ergebnis führen kann (Abb. 4, Kurve B). Dabei tritt gelegentlich eine Aufhellung des Probenleerwertes auf, die unseres Er-

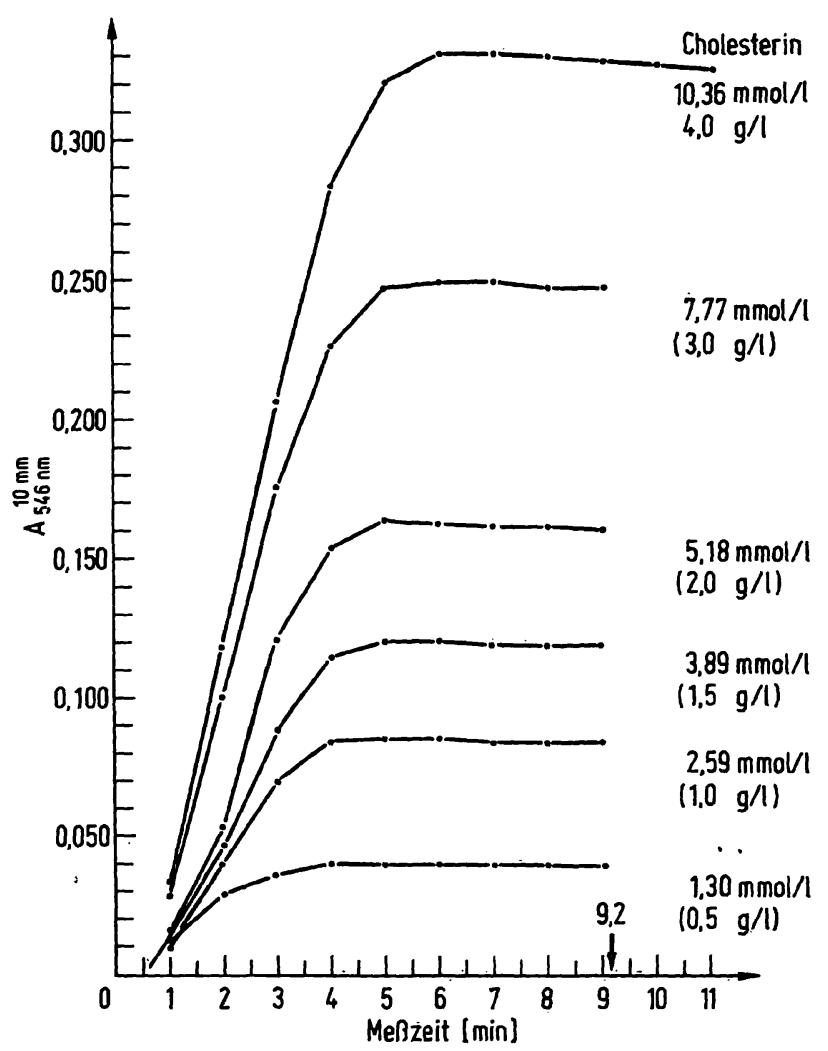

Abb. 3. Kinetik der Reaktion mit primären Standard-Lösungen (Preciset Cholesterin).

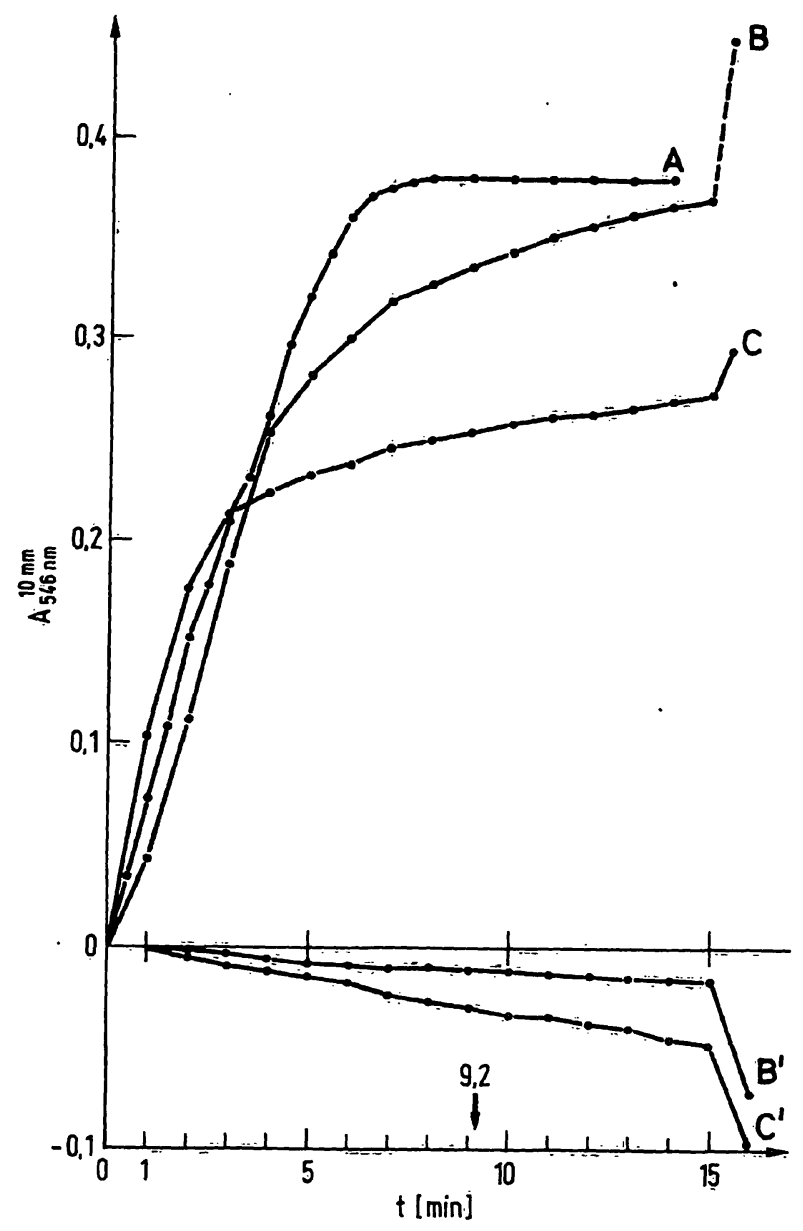

Abb. 4. Verlauf der Reaktion mit Patienten-Seren und Kontroll-Material.

GSA II-Meßzeit $=9,2 \mathrm{~min}$

A Typisches Patientenserum. Cholesterin $10,4 \mathrm{mmol} / \mathrm{l}$ $(4,03 \mathrm{~g} / \mathrm{l})$. Keine Änderung des Leerwertes.

B Extrem milchig-trübes Serum. Cholesterin etwa etwa $13,6 \mathrm{mmol} / 1(5,23 \mathrm{~g} / 1)$.

Die Kurve B gibt die Absorptionsdifferenz Hauptwert-Leerwert wieder.

B' Verlauf der Aufhellung des Probenleerwertes zu B. Kein Ende der Reaktionen B und B' nach 60 Minuten.

C Atypischer Verlauf mit einem gefriergetrockneten Kontrollserum (Sollwert 12,95 mmol/l $=5,0 \mathrm{~g} / \mathrm{l}$ ) Die Kurve $\mathrm{C}$ gibt die Absorptionsdifferenz Hauptwert-Leerwert wieder.

$C^{\prime}$ Verlauf der Aufhellung des Probenleerwertes żu C. Kein Ende der Reaktion nach 26 Minuten. Die Absorptionsdifferenz bei $9,2 \mathrm{~min}$ entspricht $7,6 \mathrm{mmol} / 1$ Cholesterin $(2,95 \mathrm{~g} / 1)$ und bei 26 Minuten $8,9 \mathrm{mmol} / \mathrm{l}$ $(3,45 \mathrm{~g} / \mathrm{l})$.

achtens auf die Wirkung der Cholesterinesterase zurück. zuführen ist. Verdünnt man derartige Proben vor der Analyse, so läuft die Reaktion mit normaler Geschwindigkeit ab.

Eine Anzahl von Kontrollseren zeigt ein identisches kinetisches Verhalten. Die nachfolgenden Täbellen (Tab. 2 und 4 ) enthalten Hinweise auf geeignetes Kontrollmaterial. 
Tab. 2. Cholesterin im Serum. PAP-Methode; Richtigkeit, langfristige Präzision und Stabilität des Faktors.

\begin{tabular}{|c|c|c|c|c|c|c|}
\hline Material & $\begin{array}{l}\mathrm{N}- \\
\text { total }\end{array}$ & $\begin{array}{l}\text { Sollwert } \\
{[\mathrm{mmol} / \mathrm{l}]} \\
(\mathrm{g} / \mathrm{l})\end{array}$ & $\begin{array}{l}\text { Istwer } \\
\text { Immo }\end{array}$ & $\begin{array}{l}1 / 11 \\
(g / 1)\end{array}$ & $\begin{array}{l}\Delta \overline{\mathrm{x}} \\
{[\%]}\end{array}$ & $\begin{array}{l}\text { VK } \\
\text { [\%] }\end{array}$ \\
\hline $\begin{array}{l}\text { Seronorm } 126 \\
\text { Pathonorm A } 11 \\
\text { Pathonorm B11 } \\
\text { Precilip } 321\end{array}$ & $\begin{array}{l}258 \\
274 \\
239 \\
267\end{array}$ & $\begin{array}{ll}2,15 & (0,83) \\
3,96 & (1,53) \\
2,54 & (0,98) \\
3,50 & (1,35)\end{array}$ & $\begin{array}{l}2,25 \\
3,73 \\
2,23 \\
3,47\end{array}$ & $\begin{array}{l}(0,87) \\
(1,44) \\
(0,86) \\
(1,34)\end{array}$ & $\begin{array}{l}+\quad 4,9 \\
-\quad 5,9 \\
-12,2 \\
-\quad 0,7\end{array}$ & $\begin{array}{l}2,8 \\
2,0 \\
2,7 \\
2,0\end{array}$ \\
\hline $\begin{array}{l}\text { Precimat } \\
\text { Cholesterin }\end{array}$ & 318 & $5,18 \quad(2,00)$ & 5,26 & $(2,03)$ & $+1,5$ & 1,9 \\
\hline
\end{tabular}

Tab. 3. Spektrale Eigenschaften des „Trinder"-Farbstoffes bei verschiedenen Reaktionspartnern. Die Reaktionen wurden mit jeweils $4,0 \mathrm{~g} / 1$ Cholesterin-Preciset $(=10,36 \mathrm{mmol} / \mathrm{l})$ unter den angegebenen Standardbedingungen für den GSA II, d. h. bei $\mathrm{pH}=7,2$, durchgeführt.

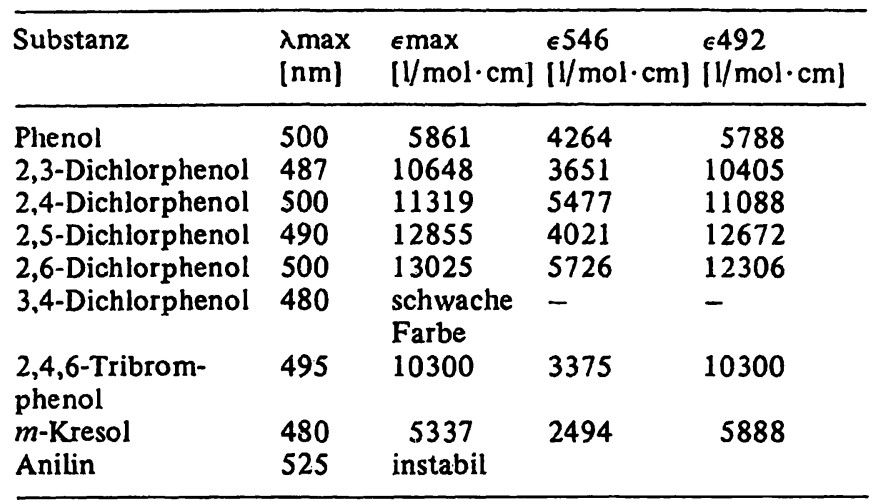

Tab. 4. Ergebnisse von Richtigkeits-Prüfungen mit dem manuellen Verfahren. Jeder angegebene Istwert ist das Mittel von 3 Bestimmungen.

\begin{tabular}{|c|c|c|c|}
\hline $\begin{array}{l}\text { Methode } \\
\text { Material }\end{array}$ & $\begin{array}{l}\text { Sollwert } \\
{[\mathrm{mmol} / \mathrm{l}]} \\
(\mathrm{g} / \mathrm{l})\end{array}$ & $\begin{array}{l}\text { Roeschlau } \\
\text { et al. (5) } \\
\text { Istwert } \\
\begin{array}{r}\text { [mmol/l] } \\
(\mathrm{g} / \mathrm{l})\end{array}\end{array}$ & $\begin{array}{l}\text { manuelles } \\
\text { Verfahren } \\
\text { Istwert } \\
\text { [mmol/l] } \\
\quad(\mathrm{g} / \mathrm{l})\end{array}$ \\
\hline $\begin{array}{l}\text { Precimat Cholesterin } \\
\text { Pathonorm B11 } \\
\text { Pathonorm A11 } \\
\text { Precilip 401B } \\
\text { Precilip 434A } \\
\text { Liponorm 50 } \\
\text { Seronorm } 126\end{array}$ & $\begin{array}{ll}5,18 & (2,0) \\
2,54 & (0,98) \\
4,48 & (1,73) \\
3,76 & (1,45) \\
3,89 & (1,50) \\
6,48 & (2,50) \\
2,07-3,00 \\
(0,80-1,16)\end{array}$ & $\begin{array}{ll}\overline{2}, 25 & (0,87) \\
3,83 & (1,48) \\
3,76 & (1,45) \\
3,89 & (1,50) \\
5,98 & (2,31) \\
2,12 & (0,82)\end{array}$ & $\begin{array}{ll}5,18 & (2,0) \\
2,20 & (0,85) \\
3,68 & (1,42) \\
3,73 & (1,44) \\
3,81 & (1,47) \\
5,80 & (2,24) \\
2,18 & (0,84)\end{array}$ \\
\hline $\begin{array}{l}\text { Lipokontroll } 017408 \\
\text { Cholesterol CTD } 317\end{array}$ & $\begin{aligned} 4,64 & (1,79) \\
10,62 & (4,10)\end{aligned}$ & $\begin{array}{ll}4,27 & (1,65) \\
9,38 & (3,62)\end{array}$ & $\begin{array}{l}4,12(1,59) \\
9,40(3,63)\end{array}$ \\
\hline
\end{tabular}

Andere Kontrollseren, zumeist solche mit starker Trübung, zeigen ein stark atypisches Verhalten (Abb. 4, Kurve C): Die Farbreaktion kann bis 60 Minuten dauern. Weiterhin werden starke Aufhellungs-Reaktionen beobachtet.

Die Reaktionstemperatur hat einen sehr deutlichen Einfluß auf den Reaktionsverlauf (Abb. 6): Bei $25^{\circ} \mathrm{C}$ wird das Maximum wesentlich langsamer erreicht als bei höheren Temperaturen. Die Maxima sind in den Grenzen der Präzision manueller Technik gleich.

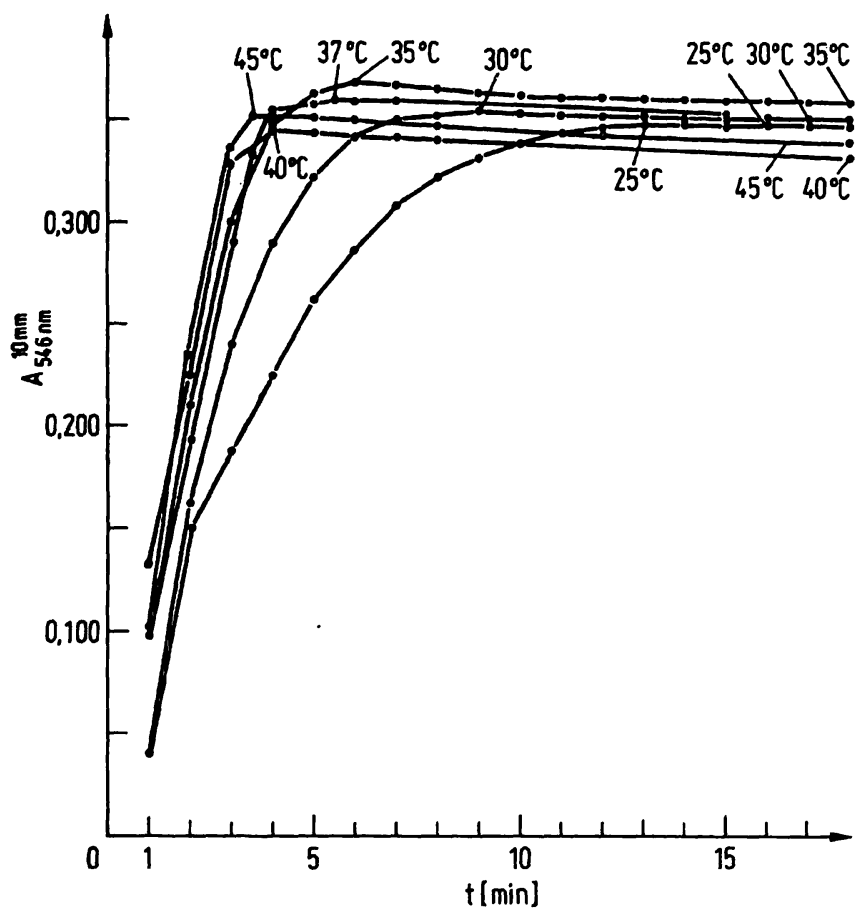

Abb. 5. Temperaturabhängigkeit der Cholesterinoxidase-Reaktion. Probe: $10,4 \mathrm{mmol} / 1$ Cholesterin (Preciset). Standard-Ansatz.

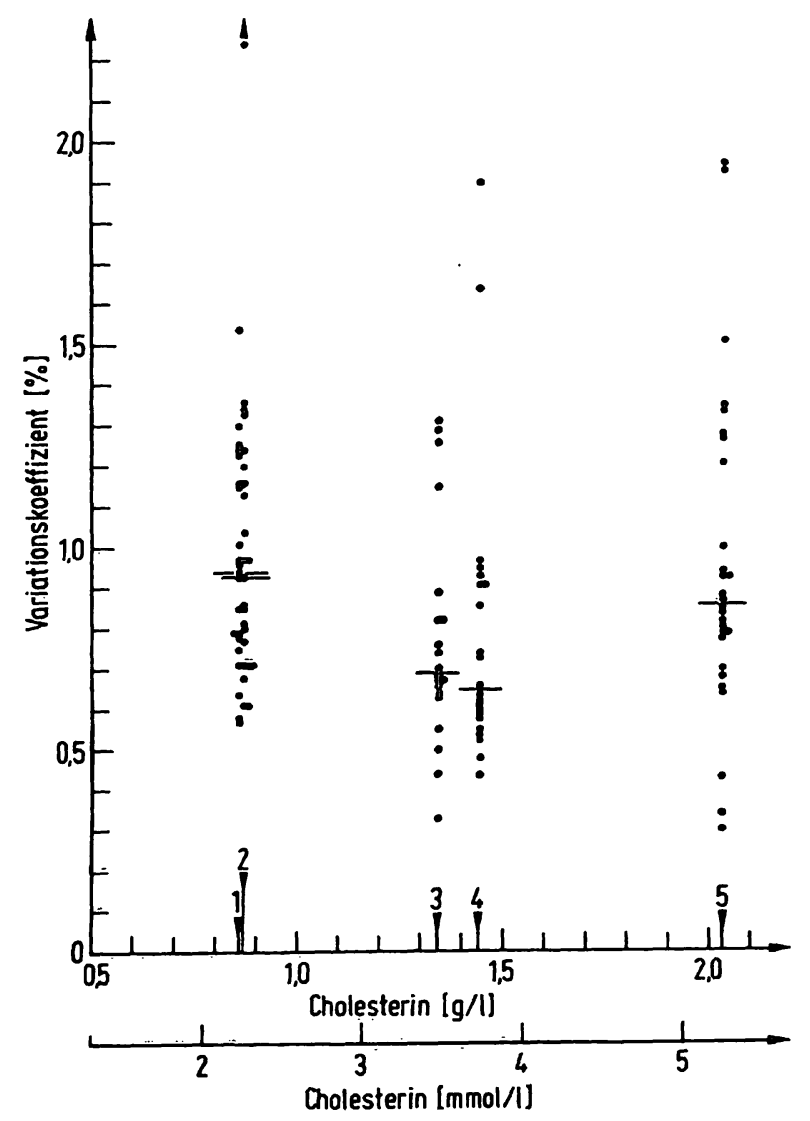

Abb. 6. Präzision in Seric.

1: Seronorm 126 (28 Serien),

2: Pathonorm B 11 (21 Serien),

3: Precilip 321 A/B (20 Serien),

4: Pathonorm A 11 (23 Serien),

5: Precimat (28 Serien).

Jeder Punkt stellt den Mittelwert einer Serie mit mindestens 8, maximal 20 Einzelwerten dar. Der Median der Gruppen ist durch einen Querstrich angedeutet. 
Im Prinzip sind Temperaturen zwischen $30-45^{\circ} \mathrm{C}$ mit Maxima von 9 bis 4 Minuten für den GSA-II möglich. Geringfügige Temperaturschwankungen $\left( \pm 0,2^{\circ} \mathrm{C}\right)$ sind in diesem Bereich unkritisch.

Eine Vergleichsuntersuchung mit verdoppelter Konzentration von Cholesterinesterase und Peroxidase ergab bei 48 Patientenseren keinen signifikanten Unterschied.

Das für das manuelle Verfahren angegebene Reagenz zeigt eine etwas andere Kinetik: Das Farbmaximum wird später, nach etwa 9-11 Minuten, erreicht. Die Farbe ist jedoch länger als 60 Minuten stabil. (Die experimentellen Details sind nicht angegeben.)

\section{Stabilität des Faktors}

Der Kalibrierungs-Faktor betrug 32,7 (mmol/l) bzw. 12,6 (g/1). Der Faktor wurde mit Precimat täglich überprüft (Tab. 2) und war 4 Monate lang stabil. Der Variationskoeffizient belief sich in 3 Monaten auf $1,9 \%$ bei einer Konzentration von 5,2 mmol/1. Die GesamtAbweichung der Werte von der Erstbestimmung betrug $+1,5 \%$. Der Absorptionskoeffizient der AutomatenMethode bei $\mathrm{pH}=7,2$ liegt etwas niedriger (etwa 86\%) als der Wert der Handmethode.

Der für die manuelle Methode vom Hersteller angegebene Faktor 22,2 (mmol/l) bzw. 8,58 (g/l) konnte reproduziert werden.

\section{Präzision und Richtigkeit der GSA-II-Methode} Es wurden insgesamt 122 Serien mit 5 verschiedenen Materialien durchgeführt (Abb. 6). Die Serienlänge belief sich dabei auf 8-20 Werte. Die Mediane der Variationskoeffizienten liegen zwischen 0,5 und $1,0 \%$ der Istwerte. Nur 7 von 122 Serien haben eine Präzision, die schlechter als $1,5 \%$ ist. (Der Vollständigkeit halber muß hier hinzugefügt werden, daß die Daten um $0,7 \%$ Ausreißer bereinigt wurden, die auf Bedienungsfehler oder technische Pannen zurückzuführen waren.) Tabelle 2 enthält weiterhin Angaben über die Richtigkeitsprüfung, die sich ebenfalls über 3 Monate erstreckte. Sollwerte wurden mit der enzymatischen Methode von Roeschlau et al. (5) ermittelt und durch eigene Messungen in der Fehlerbreite der Methodik bestätigt.

\section{Störende Begleitsubstanzen}

Einige Substanzen, von denen bekannt ist, daß sie die Indikator-Reaktion stören können, wurden durch in . vitro-Zusatz zu Preciset-Cholesterin untersucht. Novaminsulfon (Novalgin) störte die Reaktion in einer Konzentration von $100 \mathrm{mg} / \mathrm{l}(0,43 \mathrm{mmol} / \mathrm{l})$ nicht. Katalase aus Rinderleber verursachte auch in extrem hohen Konzentrationen ( $6 \mathrm{MU} / \mathrm{l}$ ) keine Störung. Ascorbinsäure reduziert in unphysiologisch hohen Dosen den gebildeten Farbstoff. Wir fanden pro $1,0 \mathrm{mmol} / 1$ Ascorbin- säure einen Signalabfall von $0,84 \mathrm{mmol} / \mathrm{l}$ Cholesterin. Bei einem Serumspiegel von etwa $10 \mathrm{mg} / \mathrm{l}$ Ascorbinsäure $(0,06 \mathrm{mmol} / \mathrm{l})$ und einem hochnormalen Cholesterinspiegel von etwa $6,0 \mathrm{mmol} / 1$ beläuft sich der Signalverlust auf höchstens $1 \%$.

Spektrale Interferenzen durch extreme Bilirubin-Konzentrationen oder durch Hämolyse werden durch Mitführen eines Probenleerwertes eliminiert.

\section{Methoden-Vergleich}

Die GSA-II-Methode wurde mit der bei uns im RoutineBetrieb befindlichen manuellen enzymatischen Methode von Roeschlau et al. (5) verglichen.

386 klinische Proben ergaben folgende statistische Ergebnisse: ( $\mathrm{x}=$ Vergleich, $\mathrm{y}=\mathrm{GSA}-\mathrm{II})$

$$
\begin{array}{lll}
\text { Mittelwerte: } & \bar{x}=5,75 & (\mathrm{mmol} / \mathrm{l}) \bar{y}=6,09(\mathrm{mmol} / \mathrm{l}) \\
& \bar{x}=2,22 & (\mathrm{~g} / \mathrm{l}) \quad \bar{y}=2,35(\mathrm{~g} / \mathrm{l}) \\
\text { Korrelation: } & & \mathrm{r}=0,987 \\
\text { Regression: } & \mathrm{x}=0,916 \cdot \mathrm{y}+0,18(\mathrm{mmol} / \mathrm{l}) \\
& \mathrm{y}=1,064 \cdot \mathrm{x}-0,02(\mathrm{mmol} / \mathrm{l})
\end{array}
$$

Mittlere

Regression (8): $\quad \bar{y}=1,078 \cdot x-0,04(\mathrm{mmol} / \mathrm{l})$

Die Punktwolke (Abb. 8) zeigt keine Ausreißer. Die mittlere Regressionsgerade hat eine etwas größere Steigung als 1,0 . Wir vermuten die Ursache in der sehr viel ungenaueren Handhabung der Vergleichsmethode im Routinelabor, bei der manuell pipettiert wurde.

\section{Steigerung der Empfindlichkeit der Methode}

Wie orientierende Versuche zeigten, ist die Verwendung der sehr viel schwächeren $\mathrm{Hg}$-Linie $492 \mathrm{~nm}$ anstelle $546 \mathrm{~nm}$ möglich. Das ergibt eine Steigerung der Empfindlichkeit um etwa 39\%, da hierbei näher am Absorptionsmaximum des Farbstoffes gemessen wird.

In einer Modifikation der beschriebenen Methode wurde versucht, die Empfindlichkeit der Methode zu steigem. Dabei wurde das Phenol durch subștituierte Derivate ersetzt. Das Problem, diese Stoffe in Lösung zu bringen, wurde durch eine kleine zusätzliche Menge Methanol als Lösungsmittel gelöst. Wie ausfuhrliche kinetische Studien zeigten, verträgt das Testgemisch diesen Zusatz. Bei Substitution des Phenols mit Halogenen ändert sich das Absorptionsspektrum des Farbstoffes in charakteristischer Weise:

Die Spektren werden schmaler, die maximalen Absorptionskoeffizienten nehmen $\mathrm{zu}$ (Abb. 8) bei geringfügiger Verschiebung zu kürzeren Wellenlängen (Tab. 3). Im Fâlle des 2,5-Dichlorphenòls läßt sich die Nachweisempfindlichkeit bei der Meßwellenlänge $492 \mathrm{~nm}$ um den Faktor 2,2 steigern. Eine Reihe dẹ in Tabelle 3 ange- 


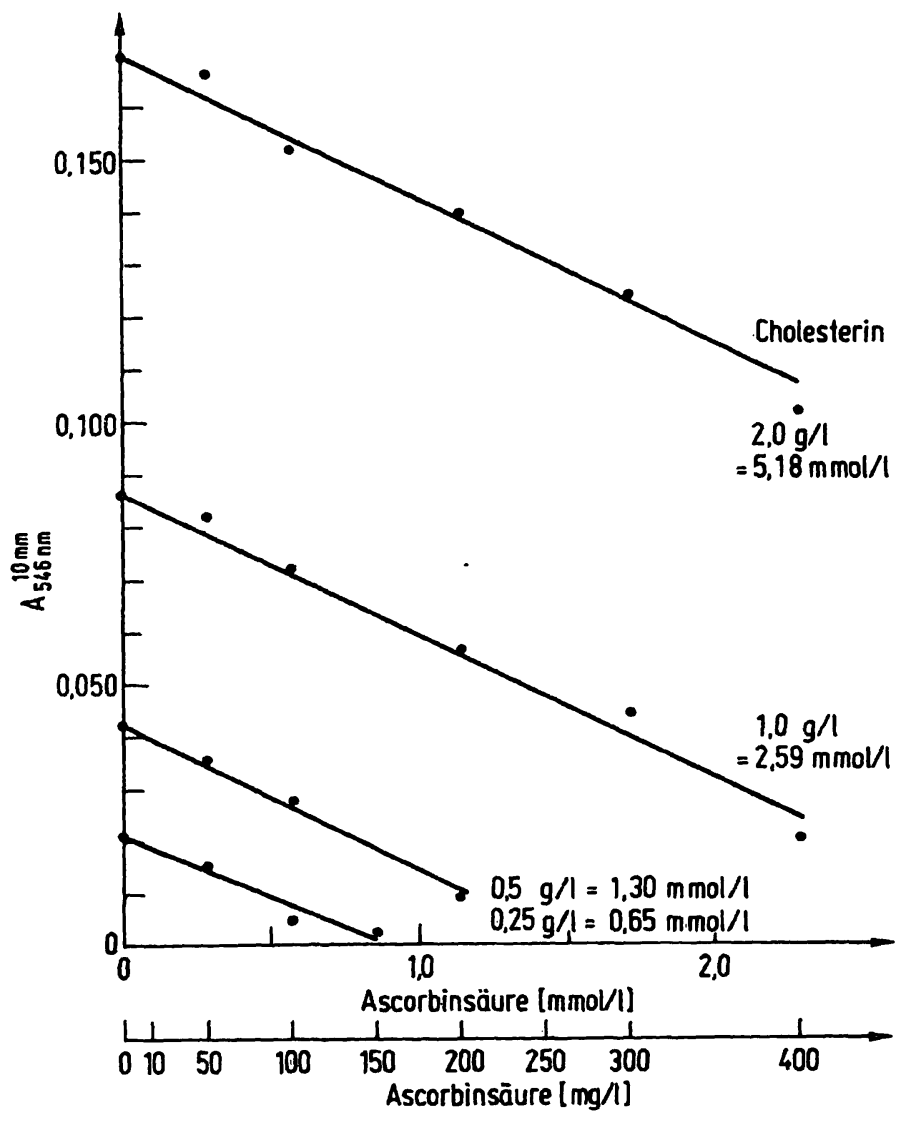

Abb. 7. Einfluß von Ascorbinsäure auf die Reaktion. CholesterinPreciset und frisch angesetzte Ascorbinsäure-Lösungen wurden $1+1$ gemischt. Jeder Punkt ist das Ergebnis einer Dreifach-Bestimmung. Der Faktor des GSA II wurde gleich 1 gesetzt, so daß das Ergebnis als Absorptionsdifferenz ausgegeben wurde.

führten Farbstoffe hat allerdings eine geringere Stabilität als das Ausgangsprodukt. Die Stabilität der 2,4-und 2,5Dichlorphenol-Farbstoffe ist jedoch ähnlich der des Phenol-Farbstoffs.

\section{Erfahrungen mit der manuellen Methode}

$\mathrm{Zu}$ Kontrollzwecken wurde zusätzlich die manuelle Methode erprobt. Tabelle 4 gibt die Ergebnisse von Richtigkeitsprüfungen weiter. Die Tabelle ergibt wieiterhin Hinweise, welchè Kontrollseren für beide Methoden geeignet sind. Die manuelle Methode lieferte nach orientierènden Versuchen befriedigende Ergebnisse.

\section{Disküssion}

Auswạh1 der Methode

Bei der Erprobung des Großautomaten GSA-II stellten wir nach 3monatigem Probebetrieb fest, daß keine der bereits adaptierten Methoden zur Bestimmung des

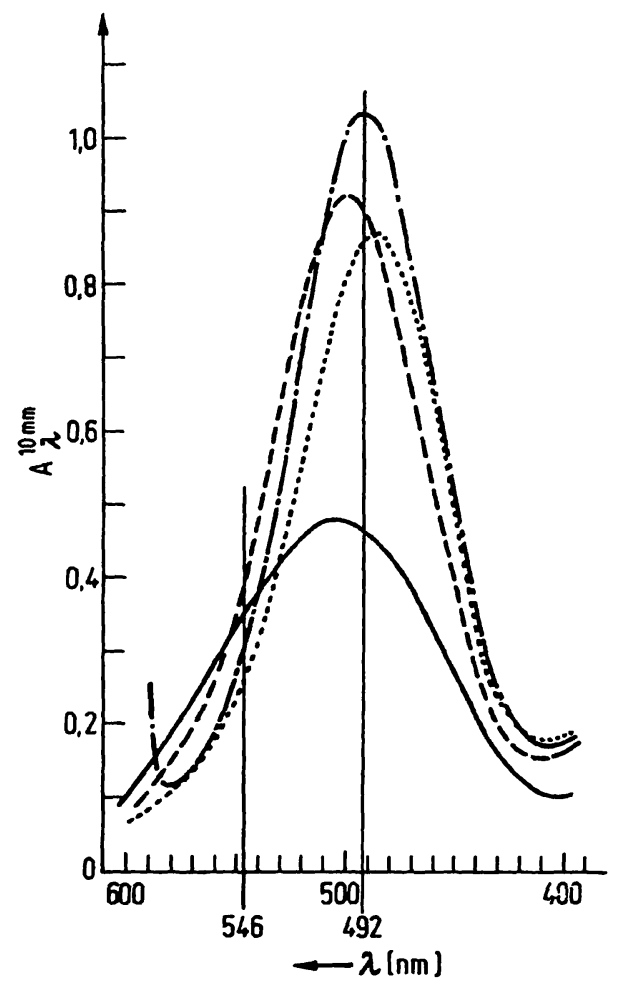

Abb. 8. Spektren von Farbstoffen mit verschiedenen Phenolen. Als Substrat diente Cholesterin-Preciset. Es wurden die Absorptionsdifferenzen zwischen Hauptwert und Reagenzienleerwert registricrt.

$\begin{array}{ll} & \text { Phenol } \\ \text {-...- 2,5-Dichlorphenol } & \text { 2,4-Dichlorphenol } \\ \ldots-\ldots- & \text { 2,3-Dichlorphenol }\end{array}$

Gesamtcholesterins zuverlässige Ergebnisse lieferte: Die klassischen chemischen Methoden, basierend auf der Liebermann-Burchardt-Reaktion, sind wenig spezifisch und arbeiten mit sehr aggressiven Reagenzien, was bei kleinen Pannen sehr leicht zu schweren Schäden am Gerät führen würde. Wir testeten die Adaptation der Methode von Roeschlau et al. (5) unter Berücksichtigung der Erfahrung von Ziegenhorn an einem GSA-II Modell (10) nach der von Jaag \& Küffer erarbeiteten Arbeitsvorschrift. Leider ergab auch diese enzymatische Methode, die sich als manuelle Methode in unserem Routine-Labor sehr bewährt hat, stark schwankende Ergebnisse im GSA-II: Der Faktor mußte häufig geändert werden. Dennoch waren die Ergebnisse der Richtigkeitsprüfung unbefriedigend. Die Hauptschwierigkeit dieser Methode liegt wohl in der langen Inkubationszeit von 70 Minuten für die nichtenzymatische Bildung des Lutidins. Im GSA-II wurden nur $45-55 \%$ des Endsignals erreicht. Die Bedingungen für eine kinetische Analyse nach dem fixed time intervall Verfahren sind offenbar über einen längeren Zeitraum schwierig einzuhalten.

Dagegen halten wir die von uns beschriebene Methode, die zuerst von Allain et al. (2) für den ABA-100 der Fa. 
Abbott, und von Klose für den Autoanalyzer (3) angegeben wurde, für ausgesprochen automaten-freundlich, zumindestens für diskrete Systeme, bei lediglich etwas geringerer Spezifität, als sie die Methode von Roeschlau (5) aufweist. Bei Beachten einiger kritischer Punkte dürfte die Anpassung an andere vollmechanische Geräte relativ einfach sein. Beim Abfassen des Manuskriptes wurden uns Adaptionen der Methode an die AnalysenGeräte Centrifichem, Fa. Union Carbide (11), Vickers 300 (12) und Perkin Elmer C 4B (13) bekannt.

\section{Qualität des Reagenz}

Das Reagenz erfült einige Anforderungen, die u. E. spezifisch für Großge räte sind: Es ist bei Kühlschranktemperatur chemisch stabil, gut löslich und behält über 1 Woche lang seine Aktivität. Die bei anderen proteinhaltigen Reagenzien häufige Zersetzung durch Mikroorganismen wurde nicht beobachtet, vermutlich ein Nebeneffekt des Phenols. Die Lichtempfindlichkeit des Reagenz ist ein Nachteil, der durch vorsichtige Hantierung jedoch kompensierbar ist. Günstig ist die Abpackung des Reagenz in Teilkomponenten, so daß eine Anpassung an gerätespezifische Größen wie z. B. Volumina möglich ist. Vor ungeprüften Veränderungen der Ionenstärke des Puffers, der Netzmittel-Konzentration und Erhöhung des Methanolanteils ist zu warnen. Bei Änderung der Substratmenge ist unbedingt zu prüfen, ob die Enzymkonzentrationen ausreichen.

Linearität der Bezugskurve, Stabilität des Faktors

Die Mehrzahl der von uns mit primären StandardLösungen erstellten Bezugskurven war bis zu 10,4 $\mathrm{mmol} / \mathrm{l}(4,06 \mathrm{~g} / \mathrm{l})$ Cholesterin linear. Gelegentlich traten jedoch Aufwärtskrümmungen der Bezugskurve in der oberen Hälfte auf, mit maximalen Abweichungen bis zu $7 \%$ von der Geraden durch den Punkt $5,18 \mathrm{mmol} / \mathrm{l}$ $(2,0 \mathrm{~g} / 1)$. Diese Beobachtung können wir trotz einer Reihe von Versuchen nur teilweise erklären. Diese Nichtlinearität gibt Trinder übrigens auch in seiner Publikation über die Glucose-Bestimmung ((4), Tab. 1) ausdrücklich ohne Erklärung an. Bemerkenswerterweise waren in allen 5 Verdünnungsreihen von Patientenseren die Konzentrations-Signal-Proportionen bis mindestens $9 \mathrm{mmol} / 1$ $(3,51 \mathrm{~g} / \mathrm{l})$, zum Teil darüberhinaus linear (vgl. Abb. 2). Die in ihrer Zusammensetzung nicht genau bekannten primären Standard-Lösungen der Preciset-Reihe vẹrdunsten etwas schneller als Wasser. Der Effekt erklärt die Abweichung jedoch nicht quantitativ.

Das günstigste Meßverfahren für den GSA-II ist die Absolutmessung. Die hier beschriebene Methode ist dafür geeignet. Der Faktor war bei täglicher Kontrolle monatelang stabil (Tab. 2). Er bezieht sich auf die Konzentration $5,18 \mathrm{mmol} / \mathrm{l}(2,0 \mathrm{~g} / \mathrm{l})$.

\section{Kinetik der Reaktion}

Die exakten Versuchsbedingungen während der Inkubation im GSA-II sind mit anderen Meßgeräten nur teilweise reproduzierbar. Umgekehrt ist ein Reaktionsablauf im GSA-II selbst nur mit viel Aufwand rekonstruierbar. Das ständige Durchmischen des Inkubates könnte die Grenzfläche zwischen wäßriger und nicht-wäßriger Phase vergrößern. Außerdem wird das Reaktionsgemisch ständig mit Luft-Sauerstoff gesättigt. Beide Umstände dürften den ersten und zweiten Reaktionșschritt, die die Gesamt-Reaktion begrenzen, beschleunigen. Dagegen dauert das Aufwärmen des Reagenz von der DosiererTemperatur $\left(4^{\circ} \mathrm{C}\right)$ auf die Inkubationstemperatur von $37^{\circ} \mathrm{C}$ etwa $1 \mathrm{~min}$. Diese Zeit muß man also hinzurechnen, wenn man das Intervall bis zur maximalen Farbausbeute aus den Kinetiken der Abbildung 3 ableiten will. Wie weit der erste Reaktionsschritt, die Esterasereaktion, zusätzliche Inkubationszeit erfordert, wurde durch Prüfen des Reaktionsverlaufs von Patientenseren im hochmolaren und mäßig erhöhten Bereich geprüft (Abb. 4). Verzögerte Reaktionen treten nur sëhr selten bei stark getrübten Seren auf, deren Cholesteringehalt auch außerhalb des Meßbereichs lag. Diese Störung ist bei genügender Aufmerksamkeit des Bedieners erkennbar. Verdünnt man solche Seren, ist die Reaktion in der vorgegebenen Zeit abgeschlossen.

Zwischen $35^{\circ}$ und $45^{\circ} \mathrm{C}$ hat die Temperatur keinen Einfluß auf die Höhe des Endsignals (Abb. 5), jedoch tritt bei höheren Temperaturen eine merkliche Beschleunigung, d. h. Verkürzung der erforderlichen Reaktionszeit auf. Temperaturschwankungen um einige Zehntelgrade sind unproblematisch. Einen zusätzlichen Vorgang bemerkten wir beim Studieren der Kinetik der Reaktion. Lipidreiche Seren sind besonders trübe. Diese Effekte verstärken sich noch durch Gefriertrocknen und Wiederauflösen, etwa bei Kontrollmaterial (14). Solche Proben werden sowohl im Hauptansatz wie im Leerwert-Ansatz im Verlauf der Reaktion klarer bis zur völligen Transparenz (Abb. 4, Kurve $B^{\prime}$ und $C^{\prime}$ ). Die eigentliche Farbentwicklung im Hauptansatz wird durch diese Aufhellungsreaktion überlagert. Die Messung sollte erst erfolgen, wenn beide Reaktionen abgeschlossen sind. Deshalb sollte auch der Dispenser für den Probenleerwert eine möglichst hohe Position haben, d. h. lange Inkubationszeit erlauben. Seren mit normalen Triglycerid- und Cholesterin-Werten zeigen in der Regel keine Aufhellungs-Reaktion. Ein kleiner Pröbenleerwert findet sich allerdings auch bei ihnen.

\section{Kontrollm aterial}

Die Eignung von Kontrollmaterial sollte auf jeden Fall dụrch Prüfung der Kinetik und durch Referenz-Messung überprüft werden. Wir haben sowohl extrem verzögerte Aufhellungsreaktionen wie langsame Farbentwick: lungen beobachtet (Abb. 4). Die Spezifität der Cholesterinesterase (vgl. 5)) schließt den Gebrauch von Kon- 
trollseren aus, die mit Cholesterinacetat o. ä. angereichert sind. Auch das Hemisuccinat, das wir nach 1. c. (7) synthe tisierten, ergab keine Reaktion.

\section{Präzision und Richtigkeit}

Eliminiert man die durch technische Pannen oder Bedienungsfehler bedingten groben Fehler, so ergibt sich eine u. E. vorzügliche Präzision sowohl in Serie als auch von Tag zu Tag. Die Ergebnisse sind wesentlich besser als die mit der manuellen Methode nach Roeschlau erzielten. Die Prüfung der Richtigkeit der Methode leidet etwas unter dem Mangel eines anerkannten und praktikablen Referenz-Verfahrens. Am besten stimmen die Ergebnisse der vorliegenden Methode mit denen der Methode von Roeschlau (5) überein. Unter diesem Vorbehalt möchten wir die Ergebnisse der Richtigkeitsprüfung als befriedigend bezeichnen.

\section{Störungen, Matrixeffekte}

Trinder (4) schlägt für sein Indikator-Reagenz den $\mathrm{Zu}$ sat $\mathrm{z}$ von Na-Azid vor, um die konkurrierende Zersetzung von $\mathrm{H}_{2} \mathrm{O}_{2}$ durch Katalase zu hemmen. $\mathrm{Zu}$ unserer Überraschung wurde die Reaktion durch Zusatz von Katalase zum Cholesterin-Standard nicht gestört. Es fragt sich, ob Na-Azid wirklich benötigt wird.

Die Peroxidase der Indikator-Reaktion ist ein wenig spezifisches Enzym (15). Die Störung durch Medikamente kann zu falsch niedrigen Ergebnissen führen (6). Besonders Novaminsulfon (Novalgin) ergibt in vivo störende Abbauprodukte, die allerdings nur bei sehr hoher Dosierung quantitativ in Gewicht fallen dürften. In vitro fanden wir mit Novalgin in hoher Konzentration keine Störung. Die etwas geringere Spezifität der beschriebenen Methode im Vergleich zur Methode von Roeschlau (5) bleibt zu beachten.

Ascorbinsäure verhindert die Farbreaktion bei Zusatz zur Probe (Abb. 7). Auch nachträglich zugese tzte Ascorbinsäure löscht die Farbe aus. Die im Serum vorkommenden Mengen sind jedoch so gering im Verhältnis zum Cholesterin, daß nur bei extrem hoher Verabreichung zu therapeutischen Zwecken störende Konzentrationen erreicht werden.

Mögliche spektrale Interferenzen durch Bilirubin oder Hämoglobin, sowie Trübungen durch Paraproteine werden durch Mitführen eines Probenleerwertes korrigiert. Aus diesem Grunde halten wir das Mitführen desselben auch für unbedingt erforderlich.

\section{Methodenvergleich}

Der Vergleich mit der Routine-Methode von Roeschlau (5) ergab an über 1100 klinischen Proben eine gute Ubereinstimmung der Ergebnisse. Insbesondere treten keine unerwarteten Ausreißer auf, die einen Hinweis auf unbekannte Störungen liefern könnten. Aus datentechnischen Gründen enthält Abbildung 9 nur die zuletzt ermittelten 386 Ergebnis-Paare.

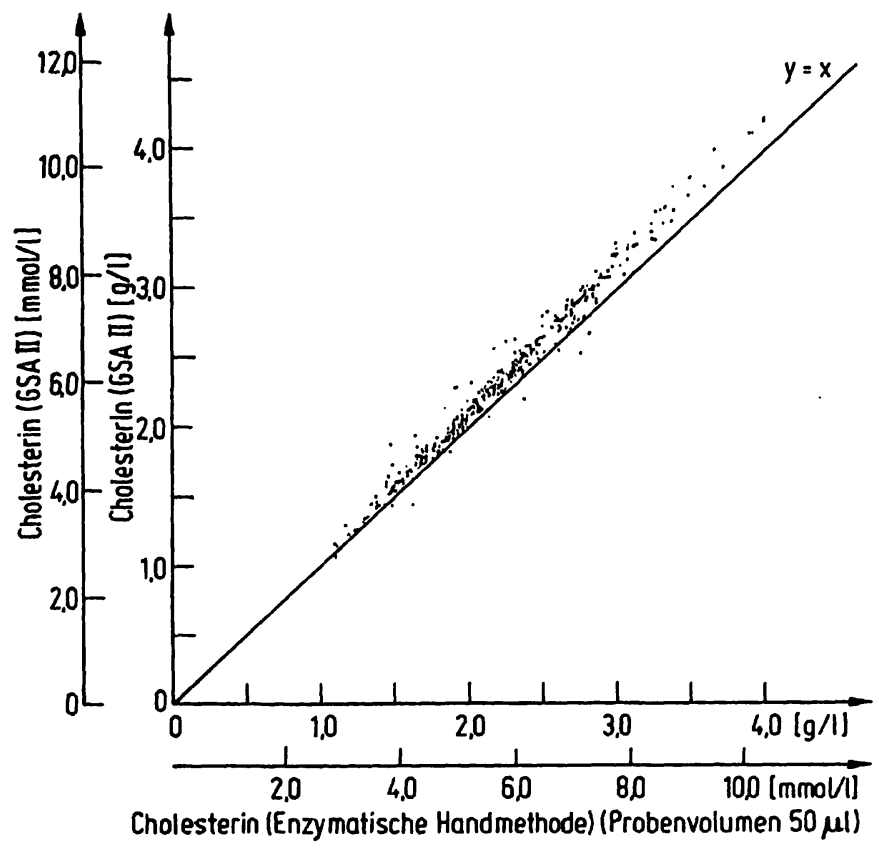

Abb. 9. Methodenvergleich mit klinischem Untersuchungsmaterial ( $n=386$ ). Abszisse: Ergebnisse der Methode von Roeschlau (5), manuelle Methode, 50 ml Probc. Ordinatc: GSA II-Methode.

Da es keinen Automaten gibt, der nicht auch einmal ausfällt, halten wir es für nützlich, für diesen Fall eine manuelle Ersatzmethode verfügbar zu haben. Deshalb haben wir die manuelle Variante der beschriebenen Methode (16) orientierend geprüf, und halten sie zu diesem Zweck für geeignet.

\section{Nach weisem p find lich keit}

Die angegebene GSA II-Methode ist fur Material von Erwachsenen mit normalen oder mäßig erhöhten Cholesterinkonzentrationen optimiert. Für andere Fragestellungen läßt sich die Empfindlichkeit durch eine andere Meßwellenlänge noch um $40 \%$ steigern.

Noch höhere Empfindlichkeiten lassen sich durch substituierte Phenole erreichen. So beschrieb Trinder die Verwendung von sulfoniertem Phenol (17) mit eınem sehr hohen Absorptionskoeffizienten von $20000 \mathrm{l} /$ $\mathrm{mol} \cdot \mathrm{cm}$, allerdings bei geringerer Stabilität des Farbproduktes. Dieses ist neben der schlechten Löslichkeit und dem stechenden Geruch das Hauptproblem der substituierten Phenole. Das von uns zur Zeit favorisierte 2,5-Dichlorphenol bietet die über doppelte Empfindlichkeit gegenüber dem jetzt benutzten Phenol. Eine ausführliche Erprobung steht jedoch aus. Wichtig ist weiterhin, daß die Farbprodukte je nach Substituent kleine Unterschiede im Absorptionsmaximum zeigen.

\section{Danksagung}

Wir danken Frau $\boldsymbol{H}$. Hartwig und Frau $E$. Borner für dic exaktc Durchführung der Versuche und die statistische Auswertung der Ergebnisse. 


\section{Literatur}

1. Greiner, R. (1973), diese Z. 11, 76-86.

2. Allain, C. C., Poon, L. S., Chan, C. S. G., Richmond, W. \& Fu, P. C. (1974), Clin. Chem. 20, 470-475:

3. Klose, S., Greif, H. \& Hagen, H. (1975), Clin. Chem. 21, 942.

4. Trinder, P. (1969), Ann. Clin. Biochem. 6, 24-27.

5. Röschlau, P., Bernt, E. \& Gruber, W. (1974), diese Z. 12, 403-407.

6. Szasz, G., Huth, K., Busch, E. W., Koller, P. U., Stähler, F. \& Vollmer, J. (1974), diese Z. 12, 256 (1974).

7. Klein, B., Kleinman, N. B. \& Foreman, J. A. (1974), Clin. Chem. 20, 482-485.

8. Averdunk, R. \& Borner, K. (1970), diese Z. 8, 263-268.

9. Stähler, F., Munz, E. \& Kattermann, R. (1970), Dtsch. Med.

10. Ziegenhorn, J. (1975), diese Z. 13, 109-115.

11. Eisenwiener, H.-G. (1975), Med. Labor. $28,81-88$.

12. Zoppi, F. \& Fenili, D. (1976), Clin. Chem. 22, 690.

13. Nennstiel, H.-J. \& Alich, R. (1976), Ärztl. Lab. 22, 185-189.

14. Proksch, G. J. \& Bondermann, D. P. (1976), Clin. Chem. $22,456-460$.

15. Bergmeyer, H. U. (1974), Methoden der enzymatischen Analyse. 3. Auflage, Verlag Chemie, Weinheim/Bergstr., S. 526.

16. Stähler, F., Gruber, W., Stinshoff, K. \& Röschlau, P.: Vortrag, Kongreß für Laboratoriumsmedizin, Berlin 21. 5. 76. 17. Barham, D. \& Trinder, P. (1972), Analyst 97, 142. Wochenschr. 100, 876-887.

Prof. Dr. K. Borner Hindenbiugdamm 30 D-1000 Berlin 45 